\title{
LAS PYMES: VÍNCULOS Y REDES DE COOPERACIÓN PARA LA INNOVACIÓN EN ANTIOQUIA (UN ESTUdio EXPLORATORIO) ${ }^{1}$
}

\author{
Gabriel Cataño Rojas* \\ Paula Botero Bermúdez ${ }^{* *}$
}

\section{Resumen:}

Este artículo presenta los principales avances de un proyecto de investigación sobre redes de innovación en pymes de la región antioqueña. La literatura internacional sostiene que la cooperación es la mejor estrategia de la que disponen las pymes en la actualidad para enfrentar su debilidad intrínseca y su aislamiento de los circuitos locales, regionales, nacionales e internacionales de mercado. Los hallazgos preliminares de una encuesta aplicada a una muestra de setenta pymes, muestran que mientras las empresas realizan actividades de innovación tecnológica y no tecnológica, y obtienen resultados positivos, la cooperación con otros agentes, empresariales, académicos y sociales, es una estrategia competitiva relativamente poco utilizada. Sin embargo, además, de una manera complementaria a la encuesta, un estudio de caso indica que existen algunos indicios de que en la región se comienzan a generar condiciones favorables para que en el largo plazo se extiendan relaciones de cooperación multiagente, que podrían dar lugar a un sistema regional de innovación más robusto y dinámico, capaz de jalonar el desarrollo económico y social.

1 Este artículo es producto de un proyecto de investigación titulado "Cooperación para la innovación en Antioquia-Colombia. Una aproximación desde la Pyme”, elaborado por los autores en el marco del proyecto "Mecanismos e Instrumentos para la Gestión Social del Conocimiento. Una perspectiva comparada: El País Vasco y Antioquia", dentro del convenio marco ITM-UPV/EHU.

- Sociólogo, UPB. Magíster en Desarrollo Social, UPB. Director del Centro de Estudios Ciudad de Medellín, ITM.

** Antropóloga, UdeA. Magíster en Estudios Urbano-Regionales, UNal. Investigadora del Centro de Estudios Ciudad de Medellín, ITM. 


\section{Palaluras claves}

Innovación tecnológica, innovación no-tecnológica, redes de innovación, redes de conocimiento, cooperación, sistema regional de innovación.

\section{Abstract}

This article is a summary of a research report in which the first results of an exploratory work about the existence of innovation networks, as networks of knowledge in Antioquian Pymes, are presented. It is widely maintained by the international literature on the subject that cooperation is the best strategy within the reach of Pymes at the moment in order to face their intrinsic weaknesses and their isolation within local, regional, national and international market circles. The preliminary findings of a survey applied to a Pymes sample show that while companies carry out technological and non technological innovation activities and obtain results, cooperation with other entrepreneurial, academic and social agents is a competitive activity seldom used. On the other hand, a case study shows that there are some regional indicators that show the emergence of favourable conditions for the generalization of multi-agent cooperation relationships which could in turn open the way to a regional system of innovation that would be more dynamic and able to carry out further social and economic development.

\section{Wey warts}

Technological innovation, not-technological innovation, networks of innovation, networks of knowledge, cooperation, and regional innovation system. 
"Continuous [...] innovations are prerequisites for sustainable competitiveness of both nations and regions. How such innovations are created and how successful innovation processes can be initiated are therefore extremely important questions. In recent years, it has been recognized that innovations are normally the result of ongoing and prolonged collaboration and interaction between firms and a variety of actors around them. These actors include customers, producers, subcontractors, consultants, public organizations, research institutes and universities, etc. Also, institutions interpreted as normative structures and "rules of the game" are given an important role in promoting stable and efficient interaction and collaboration. Against this background, a systemic approach is argued to be most appropriate. An innovation system can in principle be described as the system in which the relevant factors (i.e. actors and institutions) in an innovation process interact". Andersson y Karlsson, (2004) ${ }^{2}$.

"Lo que se mide no es siempre importante, y lo que es importante no siempre se puede medir, pero si no medimos no sabemos". Albert Einstein (Relativitäs theorie, 1926).

\section{INTRODUCCIÓN}

Es una pregunta compleja la que rige el estudio que da origen a este artículo, a saber: ¿cuándo, cómo y por qué surgen redes

2 "Las innovaciones continuas [...] son requisitos previos para la competitividad sostenible de naciones y regiones. Cómo se crean tales innovaciones y cómo pueden ser iniciados procesos exitosos de innovación son por lo tanto cuestiones extremadamente importantes. En años recientes, se ha reconocido que las innovaciones son normalmente el resultado de la colaboración e interacción permanente y prolongada entre empresas y una variedad de agentes alrededor de ellas. Estos agentes incluyen clientes, productores, subcontratistas, consultores, organizaciones públicas, institutos y universidades intensivas en investigación, etc. También, instituciones interpretadas como estructuras normativas y 'reglas de juego' están otorgando un importante papel a la promoción de la interacción y la colaboración estable y eficiente. En este contexto se presenta un acercamiento sistémico por ser el más apropiado. Un sistema de innovación puede en principio ser descrito como el sistema en el cual los factores relevantes (es decir, los agentes y las instituciones) interactúan en un proceso de innovación". Andersson y Karlsson (2004, P. 2-3) (T.de A). 
de cooperación para la innovación, entre agentes heterogéneos arraigados en un territorio $y$, particularmente, entre las pequeñas y medianas empresas y otras organizaciones de su entorno geográfico?

Estudios recientes sostienen que no existe en la actualidad evidencia concluyente que permita afirmar una relación de causalidad directa entre la innovación tecnológica y no-tecnológica y la conformación de redes de cooperación multiagente, si bien, por otra parte, otra evidencia indica que, en todo caso, las relaciones de colaboración entre firmas, independientemente de su tamaño, sector y nivel de complejidad tecnológica, y las instituciones del conocimiento, universitarias o no, favorecen en el largo plazo el éxito innovador de las firmas, y generan externalidades positivas para los territorios en los que éstas se localizan (Sebastián: 2000a; Casas et al.: 2001; Iorio: 2003; Fritsch: 2004) ${ }^{3}$

Partiendo de estos antecedentes, el principal interés de este artículo es presentar y analizar algunos datos obtenidos en un estudio empírico sobre las relaciones de intercambio y colaboración entre pequeñas y medianas empresas, y otros agentes sociales y académicos, en la subregión central de Antioquia (valle del Aburrá), relaciones tejidas en torno a proyectos y resultados de innovación tecnológica y no-tecnológica, y que pretendía identificar los patrones que siguen dichas relaciones.

El artículo se compone de la siguiente manera, a continuación de esta introducción, en la primera sección, se expone el marcoconceptual básico utilizado en el estudio; en la sección segunda, se presenta la metodología seguida y se recogen los principales

3 Sobre la base de la comparación de once regiones europeas, Fritsch (2004, 844) concluye que "Los resultados de un análisis de correlación simple no proporcionan ninguna ayuda para la sugerencia de que la cooperación o una actitud cooperativa relativamente pronunciada en una región son conducentes a la actividad de innovación [...] Si, por lo tanto, incluso un análisis más sofisticado no revelara una relación positivamente significativa entre la cooperación y la eficiencia de la $\mathrm{I}+\mathrm{D}$, éste nos dejaría con cuestiones por contestar sobre el papel de la cooperación en los sistemas de innovación. 
hallazgos del trabajo de campo, tanto el desarrollado con la aplicación de una encuesta a una muestra de setenta pequeñas y medianas empresas, como el realizado en un estudio de caso sobre una estructura de interfaz empresa-universidad-estado. Por último, se hace una síntesis, se ofrecen las conclusiones y se presenta la bibliografia.

\section{SISTEMAS REGIONALES DE INNOVACIÓN Y COOPERACIÓN: MARCO CONCEPTUAL.}

\subsection{El enfoque de sistemas innovación regionales - $\operatorname{SIR}^{3}$}

La capacidad de innovación sustentada en redes de cooperación, como mecanismo de coordinación social distinto a los mecanismos del mercado y las jerarquías, hace parte del acervo de los recursos de un territorio, de una industria o de una empresa, y es una de las cuestiones que más han interesado a los estudiosos del cambio del paradigma tecno-económico en curso ${ }^{5}$.

Un enfoque útil para enfrentar el fenómeno de la cooperación y de las redes de innovación es el de los sistemas de innovación

4 Mucho se ha discutido, y se sigue discutiendo, sobre la pertinencia que tiene el concepto o el enfoque de sistemas de innovación regionales cuando se trata de aplicarlo a América Latina, puesto que se argumenta que mientras que en Europa es un concepto ex post, esto es, expresión de un fenómeno empírico previamente dado, en América Latina, es un concepto ex-ante, es decir, que si bien se puede afirmar que en el subcontinente se da innovación, esta no se produce sistemáticamente, y muchas veces ni siquiera formalmente. Sin querer eludir el debate, pero para el que no hay espacio en este artículo, baste con advertir que el uso plural del término que se hace aquí, remite a una diversidad de experiencias concretas y no implica la exhaustividad y completud que connota el mismo término usado en singular. Quizás, entonces, sea mejor hablar de "enfoque" y no de "concepto", de una manera de considerar un asunto y no de una idea abstracta o de un ideal-tipo. (Arocena $R$. y Sutz, J. 1999).

5 Pérez, 1988, 1999; Castells, 1999; Sebastián, 2000a, 2000b, 2000c; Rozga, 2003; Luna y Velasco, 2005; Martínez del Río y Céspedes-Llorente, 2006; Casanueva, Castro y Galán, 2006; Camisón, Boronat y Villar, 2005). 
regional (SIR), término del que se encuentran diversas definiciones en la literatura ${ }^{6}$. A pesar de esta diversidad, un conjunto tan amplio de definiciones tiene elementos comunes, aunque sus elementos difieran en el énfasis que ponen sobre uno u otro aspecto. Una definición general pero no universal del término (de allí el plural), consiste en definir los SIR de una variedad de maneras: ellos pueden ser nacionales, regionales, sectoriales, o tecnológicos. Todos ellos implican la creación, difusión y uso de conocimiento. Los sistemas consisten en componentes, relaciones entre estos, y sus características o atributos (Carlson et al. 2002).

De la definición anterior se subrayan los siguientes aspectos:

1. la idea de sistema, particularmente, de sistema social, que puede incluir individuos, grupos, organizaciones, instituciones, etc.;

2. la idea de interacción entre los elementos, específicamente, debido a las redes que se pueden generar;

3. los flujos que circulan a través de dichas redes, que para el caso de los sistemas de innovación, están constituidos principalmente por información y conocimiento (tácito y codificado), económicamente útiles;

4. la idea de región que alude a la existencia de un territorio económica, social, política y culturalmente construido, donde la proximidad geográfica facilita el contacto y los intercambios entre los agentes;

5. finalmente, la idea de historia, porque los sistemas de innovación evolucionan a lo largo del tiempo, a tal punto que es difícil que un sistema de innovación permanezca idéntico a sí mismo en dos momentos distintos del tiempo, modificándose sus componentes y las relaciones entre ellos.

$6 \quad$ Koschatzky, K. 2001; Asheim, B. \& Isaksen, A. 2001; Noisi, J. 2000; Eriksson, A. 2000; Meeus, M., Oerlemans, L. \& van Dijck, J. 1999; Koschatzky, K. 1998a; Koschatzky, K. 1998b; Cooke, P., Uranga M. J. \& Etxebarria, G. 1998; Cooke, P., Uranga M. J. \& Etxebarria, G. 1997; Edquist, C (Ed.). 1997; Asheim, B. \& Isaksen, A. 1996; Lundvall, B. 1995 . 
Además, en esta línea de análisis, el enfoque de SIR permite plantear que (Andersson y Karlsson: 2004):

a. los sistemas de innovación son más fácilmente observables en el nivel regional;

b. el reconocimiento por los investigadores del papel que juegan los entornos económicos y la proximidad geográfica en la innovatividad de las empresas. Las rutinas informales y las normas que son específicas a cada región juegan un papel esencial en el comportamiento de las firmas y en las formas de colaboración entre ellas;

c. el conocimiento tácito y no codificado es muy importante para los procesos de innovación porque los contactos cercanos y las relaciones cara a cara son prerrequisitos para el intercambio de este tipo de conocimiento.

Además, y dado que este artículo parte de un proyecto de investigación internacional que busca comparar dos sistemas de innovación regionales, uno "central" (el País Vasco) y otro "periférico"7 (Antioquia), es necesario tener en cuenta que los SIR tienen diversas características en diferentes regiones dependiendo de la especialización económica y del contexto institucional. Los sistemas de innovación en regiones de alta tecnología difieren, por ejemplo, de los sistemas de innovación en regiones tradicionales que se especializan en industrias basadas en recursos naturales (este sería el caso de Antioquia). Incluso, los SIR, dadas ciertas especificidades regionales, como rutinas y normas, pueden probablemente diferir aunque tengan similares estructuras económicas (Carlson et al. 2002).

En consecuencia, y teniendo presentes todos los elementos conceptuales seleccionados, otra crucial cuestión que surge es

\footnotetext{
7 'In a broad sense, a region in the 'centre' may be defined as an easily accessible location characterised by relatively high density of population and economic activity. A centre has a relatively high rank in the spatial hierarchy. In contrast, regions in the 'periphery' are lacking these properties. They are characterised by relatively low density, poor accessibility and rank relatively low." (M. Fritsch: 2004, p. 832).
} 
si es razonable esperar que regiones con una larga tradición de subdesarrollo ${ }^{8}$-como es el caso de la región antioqueña, pueden generar capacidades dinámicas ${ }^{9}$ a partir de innovaciones, las cuales tengan como estrategia central relaciones de cooperación entre diversos agentes.

En síntesis, en este artículo se entiende un ("no el") sistema de innovación regional como "un conjunto de redes de agentes públicos, privados y educacionales que interactúan en un territorio específico, aprovechando una infraestructura particular, para los propósitos de adaptar, generar y/o difundir innovaciones" (Carlson, B. \& Stankiewicz: 1991, p. 13). Los SIR pueden llevar incita la idea de redes de cooperación, a tal punto que la innovación desde un punto de vista sistémico se podría definir como un proceso y a la vez como el resultado de relaciones colaborativas entre agentes heterogéneos, que forman redes de las que se puede estudiar la morfología y la dinámica de interacción entre sus nodos.

Dado este marco en este artículo se entiende la innovación en un sentido amplio, es decir, como innovación en general compuesta por innovaciones de producto, de proceso y organizacional a nivel de

8 Arocena y Sutz (2002, p. 1) plantean el problema en los siguientes términos: “¿Cuáles son los países en desarrollo? Su heterogeneidad desafia una caracterización pero, hoy como ayer, es posible definirlos por oposición. Cuando la transición de las sociedades agrarias a las sociedades industriales enmarcaba el acontecer histórico mundial, los países "en desarrollo" fueron los que no ingresaron en la senda de la industrialización, lo cual los ubicó en posiciones poco favorables ante la expansión del Occidente industrializado, configurándolos así como países subdesarrollados más que "en desarrollo". Cuando, al presente, vivimos la transición a la sociedad del conocimiento, en los países desarrollados la economía se basa sólidamente en la ciencia, la tecnología, la innovación y la educación avanzada, mientras que países "en desarrollo" son los otros, los que no logran hacer de la generación, transmisión y uso del conocimiento un vector fundamental de crecimiento económico y progreso social".

9 En este artículo se entiende como capacidades dinámicas a aquellas capacidades que permiten la continua adaptación de una región al entorno cambiante. A su vez una capacidad es la combinación convenientemente coordinada de recursos cuya aplicación dará origen a la realización de ciertas tareas o actividades. El principal recurso al que se alude aquí es el conocimiento y la capacidad de aprender. (Hall: 1993). 
la firma, así como innovación social e institucional a nivel de una industria, de una región y de una nación (Morgan, 1997: 492). A estos tipos de innovaciones, hay que agregar, además, la innovación de marketing, que aunque no considerada explícitamente por Morgan, fue tipificada hace tiempo por Schumpeter (1934) y, recientemente, introducida en el Manual de Oslo (2005). La innovación social e institucional incluye, específicamente, las redes de conocimiento y las redes de innovación ${ }^{10}$, que constituyen el capital relacional de las empresas.

Todas estas cuestiones se tuvieron en cuenta en el diseño de la encuesta y del estudio de caso que brindan la base empírica de este trabajo.

\subsection{Cooperación, innovación y constitución de redes}

Encadenado teóricamente al concepto de sistema regional de innovación se encuentra el concepto de cooperación entre agentes en el seno de redes de innovación y entre distintas redes en un mismo sistema de referencia territorial. En efecto, más que en sus nodos y sus atributos, las redes de innovación descansan en las interacciones entre los nodos y los flujos que circulan entre ellos. Si los nodos y las redes a las que aquí se alude son, principalmente, empresas y redes de empresas del sector privado, que por definición se alinean en el mercado como principal estrategia de coordinación de su actividad económica, la pregunta que surge, entonces, es ¿porqué y cómo, tratándose de empresas, cuyo principal dictum - según la teoría económica ortodoxa - es una feroz competencia,

10 “[...] las redes de conocimiento constituyen sistemas o estructuras complejas configuradas por actores heterogéneos, que se basan en flujos de información y conocimiento y, en su caso, en la generación de nuevo conocimiento, para la solución de problemas específicos" (Luna y Velasco: 2005, p. 4). Por su parte el Forfás define las redes de innovación como "grupos independientes de instituciones y/o compañías que son: colaboradoras y competidoras; geográficamente localizadas en una o varias regiones nacionales; especializadas en un campo particular, ligado por tecnologías y habilidades comunes; basadas en la ciencia o relacionadas a negocios; formales o informales". 
puede surgir la cooperación como una estrategia de colaboración entre empresas y entre éstas y otras organizaciones sociales, que es lo que está incito en el concepto de sistema de innovación regional? Tratándose de pequeñas y medianas empresas en un contexto de subdesarrollo, la cuestión emerge con mayor fuerza, porque éstas por definición carecen de los recursos suficientes, especialmente de conocimiento e información, para realizar innovaciones demasiado complejas por sus propios medios. Si como con razón se ha dicho el problema de la pyme no es tanto su tamaño como su aislamiento, este tipo de empresas pueden entonces acceder a tales recursos conformando redes que preferentemente pueden surgir en la proximidad geográfica y cultural que depara la región (Fraunhofer Institute for Systems and Innovation Research, 1999).

En suma, se plantea así, en primer término, el problema de la relación entre innovación y tamaño organizativo. Puesto que durante mucho tiempo se creyó que la capacidad innovadora de una empresa estaba relacionada exclusivamente con su tamaño, tamaño derivado de la acumulación y centralización del capital en determinadas zonas geográficas, y en determinados sectores y firmas industriales, dado que parecía natural suponer que aquellas empresas más grandes dominaban una mayor cantidad de recursos diversos, los cuales podía destinar a actividades de innovación, se pensó que la innovación se debía explicar únicamente en función del tamaño de las empresas. Sin embargo, últimamente, cada vez más los expertos están poniendo en duda esta creencia (Camisón et al., 2003). Si en la economía del conocimiento y del aprendizaje, ya no son el capital y los recursos naturales los factores de producción par excellence, sino que es el conocimiento y la información los que ha devenido recurso estratégico, entonces, las empresas mejor preparadas para emprender innovaciones serán aquellas que puedan acceder a un mayor stock de conocimientos e informaciones, que puedan usar de manera económicamente útil, acceso que no se relacionará necesariamente con su tamaño en términos de número de empleados o monto de su patrimonio, sino en primer lugar con su capacidad de absorción de conocimiento. La capacidad 
de absorción se refiere a la capacidad de aprendizaje que tenga una empresa, la que estará determinada a su vez por la cantidad y calidad de su capital humano, lo mismo que por el éxito en la gestión del conocimiento, tanto explícito como tácito.

En consecuencia, este conjunto de argumentos llevó a que en el estudio que se presenta, tanto la naturaleza y el alcance de la I+D realizada por las pymes, como el desarrollo de otras actividades que les permiten a las empresas acceder a conocimientos útiles, hubieran sido temas que se tuvieron preferentemente en cuenta en el diseño de la encuesta y del estudio de caso.

En relación con lo anterior, las redes de cooperación constituyeron el otro gran capítulo incluido en el trabajo de investigación. Estas redes se definen como asociaciones de interesados que tienen como objetivo la consecución de resultados acordados conjuntamente a través de la participación y la colaboración mutua (Sebastián: 2000d). En el caso más específico de la innovación, la cooperación se debe entender como un vínculo entre empresas con un determinado fin que, uniendo o compartiendo gran parte de sus capacidades y/o recursos, sin llegar a fusionarse, instauran cierto grado de cooperación para realizar una o varias actividades que contribuyen a incrementar sus ventajas competitivas (Ángel del Brío et al., 2006). Además, desde un punto de vista ampliado de la innovación, también se consideraron los vínculos y las relaciones de cooperación empresa-universidad, junto con las relaciones entre las empresas y otras instituciones políticas y sociales (Sebastián: 2000d). A mayor abundamiento, a parte de las redes de cooperación empresa-universidad, también se rastreó la existencia de otros tipos de redes, distinguidas por la literatura especializada (Iorio, 2003): redes de información y de comunicación, redes académicas, redes temáticas, redes de investigación, y redes de servicios tecnológicos.

Como se verá en la siguiente sección, en el material empírico del estudio que sirve de base al presente artículo, interesó analizar si en la dinámica innovadora de las pymes antioqueñas, las redes de innovación como redes de conocimiento, están jugando o no un 
papel significativo y hasta qué punto, y si se observan otras clases de vínculos. En particular, las relaciones empresa-universidad son muy significativas para la dinámica de los sistemas de innovación, más allá de las redes industriales, pero es necesario considerar que existe una enorme diferencia en los vínculos entre las empresas y las universidades, según la etapa de evolución por la que atraviesen tales relaciones ${ }^{11}$.

Asimismo, en el caso de la cooperación empresa-empresa, son diversas las razones que llevan a las empresas a cooperar: el carácter fijo de muchos de sus costes, como la investigación y el desarrollo; el aprovechamiento de la complementariedad de sus recursos (asimetrías o sinergias); la facilidad para que las empresas compartan riesgos por varias vías; el papel auspiciante del gobierno; el aprendizaje de los adelantos tecnológicos de los socios que multiplica el potencial de cualquier tecnología; la penetración en un determinado mercado y, finalmente, la fijación de estándares de un sector en el que se desarrollan tecnologías complejas. Estas mismas ventajas -mutatis mutandis- son predicables de la cooperación en $\mathrm{I}+\mathrm{D}+\mathrm{i}$ entre pymes, y no sólo entre grandes empresas. No es de extrañar por tanto que la cooperación se haya convertido en un

11 Es plausible distinguir tres etapas en el desarrollo de las relaciones empresauniversidad, según la evolución que hayan tenido sus contenidos; la primera de ellas, denominada propiamente relaciones empresa-universidad, "se caracteriza porque se producen de una manera unidireccional sobre la base de la existencia de unos servicios en las universidades que pueden ser utilizados por las empresas como simples clientes. Una segunda etapa está definida por una relación más estrecha de oferta/demanda y se define como vinculación empresa-universidad. Las universidades no solamente ofrecen servicios estandarizados ante unos planteamientos rutinarios por parte de las empresas, sino que se establece un diálogo y una negociación frente a demandas específicas de las empresas, lo que requiere una mayor flexibilidad y capacidad de adaptación por parte de las universidades. La tercera etapa se caracteriza por una interacción basada en actividades conjuntas, en las que hay compromisos institucionales explícitos. Esta etapa se define como cooperación empresa-universidad. Supone un grado mayor de interacción, especialmente por parte de las empresas, que no son solamente clientes de las universidades sino socios de ellas en proyectos y actividades conjuntas a través de acuerdos y alianzas, así como de consorcios multiinstitucionales" (Sebastián: 2000d). 
tema clave en la literatura de los últimos años (Ángel del Brío et al., 2006), y haya sido incluida en este estudio.

En un frente convergente con el anterior, son distintos los argumentos cuando las que se consideran son las relaciones de cooperación entre empresas y universidades. En efecto, los objetivos que persiguen y la cultura social de investigación que las ha caracterizado, son muy disímiles y difíciles de armonizar. Mientras en la universidad la práctica de una ciencia abierta suele jugar el papel protagónico (amplia difusión de los resultados de la investigación a través de publicaciones sometidas al juicio crítico de pares externos), la investigación industrial está sometida a un riguroso régimen de propiedad o está protegida por el secreto. La investigación universitaria es principalmente básica, en cambio la investigación industrial es aplicada fundamentalmente. En cuanto a los hábitos sociales, el interés primordial para el investigador universitario es dar a conocer los avances de sus investigaciones, lo que le permite un reconocimiento en la comunidad científica y el fortalecimiento de sus redes personales, pues no le preocupa mucho que sus ideas sean robadas o copiadas por otros colegas. Un hábito semejante en el caso de una empresa sería un suicidio, pues daría una ventaja evidente a sus rivales, ya que estos pueden mantener bajos sus costos de producción debido a que no tendrían que incurrir en grandes inversiones. Estas diferencias generan una estructura diferente de redes personales entre universidad e industria (Iorio: 2003, pág. 7).

De novo, un interés marcado de la encuesta fue precisamente conocer sobre la existencia de esta clase de redes ancladas en pymes.

\section{InNOVACIÓN y COOPeración en ANTIOQUIA: Base eMPIRICA}

En este apartado se describe la metodología de un trabajo de campo mediante encuestas, aplicadas a setenta pymes antioqueñas,

a fines del 2006. También se presentan los objetivos del trabajo, lo mismo que algunos de los hallazgos más significativos a la luz 
del marco conceptual desarrollado. La exposición se complementa con los resultados de un estudio de caso sobre una organización de interfase, intentando mostrar cómo a partir de su constitución y evolución, se están comenzando a construir relaciones de confianza y cooperación entre empresas antioqueñas emprendedoras, un creciente y diversificado número de grupos y centros de investigación universitarios, y unos pocos centros de desarrollo tecnológico. La hipótesis empírica que se propone es que se está fraguando en Antioquia, mediante este mecanismo, un sistema de innovación regional más robusto económica y socialmente, en el largo plazo.

\subsection{Descripción ale la metodología}

Debido al relativo desconocimiento del tema en la región, se decidió realizar un estudio de carácter exploratorio, y por ello se eligió la metodología de encuestas, dado que permitía estudiar un gran número de empresas, al tiempo que examinar diversos temas de una manera general. Sin embargo, habida cuenta de la magnitud del error estándar ${ }^{12}$, se decidió, así mismo, emprender un estudio de caso sobre una estructura de interfaz universidad- empresaestado, que permitiera un cuadro más variopinto y complejo del fenómeno estudiado ${ }^{13}$. Por lo tanto, debe tenerse en cuenta que

12 "El error estándar determina la probabilidad que tiene un estimador (por ejemplo la media) de ser igual a la media estimada a partir de una muestra, empleando otra muestra. Esto es, la probabilidad de que la media muestral sea la media poblacional. Se asume que el mínimo de probabilidad aceptable de que la media muestral sea la media poblacional es $80 \% "$ (Durán et al. 2000, pág. 195).

13 Aunque en la actualidad existen ofertas económicamente muy favorables, que gracias al uso extensivo e intensivo de las nuevas tecnologías de información y comunicación, permiten una relación coste/beneficio positiva para los investigadores, y que se consideró utilizar en el estudio, la carencia en las pymes antioqueñas de una cultura en el uso de estas nuevas tecnologías, obligó a tener que contratar entrevistas personales, que por sus elevados costos unitarios y los escasos recursos financieros de los que se disponía, hicieron imposible trabajar con una muestra más amplia de empresas, y tener que operar con un error estándar de $9.3 \%$ (ver ficha técnica). Así fue que se pensó, entonces, como alternativa realizar un estudio de caso, que sirviera de medio de contraste, útil al fin y al cabo, desde el punto de vista de un estudio exploratorio (Yin: 1994). 
las conclusiones que se ofrecen al final del presente artículo, no pueden ser más que provisorias, y que sólo cuando el estudio sea completado en la fase cualitativa que se está llevando a cabo en la actualidad, se podrá ofrecer conclusiones más robustas.

Fueron dos los principales objetivos que se esperaba alcanzar con la aplicación de la encuesta, a saber: Identificar las innovaciones implementadas por las pymes antioqueñas en el periodo 2004-2005, y conocer sobre el papel desempeñado en la innovación por los vínculos y las relaciones de cooperación entre las pymes y otras empresas y/o organizaciones en el logro de innovaciones tecnológicas.

En el Cuadro $N^{\circ} 1$ se presenta la ficha técnica de la encuesta, cabe destacar cómo en la muestra extraída se representan porcentualmente las actividades manufactureras y de servicios, según una base de datos de 1.467 pymes, que cubre la subregión central de Antioquia -valle del Aburrá-, descartándose el resto de subregiones debido a que en ellas el sector manufacturero es inexistente o poco significativo ${ }^{14}$. Según el tamaño por número de empleados, en la muestra sobresale la pequeña empresa (entre 11 y 50 empleados), característica en la que se sitúan el $71,4 \%$ de las empresas, frente al $28,6 \%$ que corresponde a las empresas medianas (entre 51 y 200 empleados). Clasificadas por sector económico, el 74,3\% de las pymes de la muestra corresponden al sector industrial, mientras que el $25,7 \%$ representa al sector servicios.

Esta doble distribución muestral se justifica, primero, porque el tamaño de las empresas ha estado asociado tradicionalmente a la capacidad innovadora de éstas -a mayor tamaño mayor capacidades y recursos para la innovación-y, segundo, porque el sector de actividad económica donde las pymes se desempeñan -manufactura o servicios- está asociado al tipo y alcance de las innovaciones que realizan las empresas.

14 El valle del Aburrá aporta el 66\% del PIB de Antioquia, y dentro de éste la industria participa con el $91,2 \%$, lo que significa que basta estudiar esta subregión para tener una idea clara de lo que ocurre en el resto de la región (Atlas Estratégico de Antioquia, 2005). 


\begin{tabular}{|c|c|c|c|c|c|c|c|c|c|c|c|}
\hline \multicolumn{12}{|c|}{ CUADRO $\mathrm{N}^{\circ}$ 1: FICHA TÉCNICA DE LA ENCUESTA } \\
\hline Referencia del estudio: & \multicolumn{11}{|c|}{$\begin{array}{l}\text { Estudio explor } \\
\text { en Pymes Anti }\end{array}$} \\
\hline Naturaleza del estudio: & \multicolumn{11}{|c|}{ Cuantitativa } \\
\hline Técnica: & \multicolumn{11}{|c|}{ Entrevista personal con cuestionario est } \\
\hline Unidad de análisis: & \multicolumn{11}{|c|}{ Pymes del departamentos de Antioquia } \\
\hline $\begin{array}{l}\text { Unidades de } \\
\text { información: }\end{array}$ & \multicolumn{11}{|c|}{$\begin{array}{l}\text { Gerentes o administradores de Pymes manufactu- } \\
\text { reras o de servicios }\end{array}$} \\
\hline Marco mue & \multicolumn{11}{|c|}{$\begin{array}{l}\text { Base de datos con } 1467 \text { Pymes Antioqueñas. Sector } \\
\text { manufacturero: } 1.131 \\
\text { Sector servicios: } 336\end{array}$} \\
\hline Encuesta piloto: & \multicolumn{11}{|c|}{$\begin{array}{l}12 \text { encuestas, de las cuales las dos primeras no se } \\
\text { incluyeron en la muestra total ( } 70) \text {, dado que el } \\
\text { cuestionario fue ajustado para garantizar la calidad } \\
\text { de la información recolectada. }\end{array}$} \\
\hline \multirow{4}{*}{$\begin{array}{l}\text { Tamaño de muestra y } \\
\text { distribución } \\
\text { porcentual por sector } \\
\text { de actividad y tamaño } \\
\text { de empresa: }\end{array}$} & \multicolumn{5}{|c|}{ Actividad económica } & \multicolumn{6}{|c|}{ Tamaño de empresa } \\
\hline & Industria & \multicolumn{2}{|c|}{ Servicios } & \multicolumn{2}{|c|}{ Total } & \multicolumn{2}{|c|}{ Pequeña } & \multicolumn{2}{|c|}{ Mediana } & \multicolumn{2}{|c|}{ Total } \\
\hline & $\%$ & $\mathrm{~N}$ & $\%$ & $\mathrm{~N}$ & $\%$ & $\mathrm{~N}$ & $\%$ & $\mathrm{~N}$ & $\%$ & $\mathrm{~N}$ & $\%$ \\
\hline & 5274,3 & 18 & 25,7 & 70 & 100 & 50 & 71,4 & 20 & 28,6 & 70 & 100 \\
\hline $\begin{array}{l}\text { Nivel de confianza y } \\
\text { error muestral: }\end{array}$ & \multicolumn{11}{|c|}{$\begin{array}{l}\text { Nivel de confianza del } 95 \% \text {. Error muestral del } 9.3 \% \text {, } \\
\text { para población finita }\end{array}$} \\
\hline $\begin{array}{l}\text { Fecha del trabajo de } \\
\text { campo: }\end{array}$ & \multicolumn{11}{|c|}{ Octubre 23 a noviembre 17 del año $2006^{15}$} \\
\hline
\end{tabular}

Por otra parte, el Cuadro $\mathrm{N}^{\circ} 2$ presenta la distribución muestral según el código CIIU revisión $3^{16}$, que clasifica las empresas subsectores de actividad económica.

15 El trabajo de campo fue realizado por la empresa Consenso S.A.

16 Código Industrial Internacionall Uniforme-Revisión 3. 


\begin{tabular}{|c|c|c|c|}
\hline \multicolumn{4}{|c|}{$\begin{array}{c}\text { CUADRO Nº 2: DISTRIBUCIÓN DE LA MUESTRA SEGÚN } \\
\text { CÓDIGO CIIU-REV. } 3\end{array}$} \\
\hline Código ClIU & SUBSECTOR ECONÓMICO & $\%$ & $\mathbf{N}$ \\
\hline 150 & Alimentos y bebidas & 6 & 9,1 \\
\hline 170 & Textil & 4 & 6,1 \\
\hline 180 & confección & 10 & 15,1 \\
\hline 190 & Cuero-calzado & 3 & 4,5 \\
\hline 200 & Madera & 2 & 2,8 \\
\hline 210 & Papel & 1 & 1,5 \\
\hline 230 & Derivados petróleo & 1 & 1,5 \\
\hline 240 & Sustancias químicos & 6 & 9,1 \\
\hline 250 & Plástico & 4 & 6,1 \\
\hline 270 & Productos de hierro & 2 & 3,0 \\
\hline 280 & Productos de metal & 2 & 3,0 \\
\hline 290 & Maquinaria general & 3 & 4,3 \\
\hline 340 & Fabr. vehículos & 2 & 3,0 \\
\hline 360 & Muebles de metal & 5 & 7,1 \\
\hline 450 & Construcción & 1 & 1,5 \\
\hline 510 & Comercio & 7 & 10,0 \\
\hline 720 & Informática & 4 & 6,1 \\
\hline 740 & Otras actividades & $\begin{array}{lll}7 & & \\
\end{array}$ & 10,0 \\
\hline \multicolumn{2}{|l|}{ TOTAL } & 70 & 100 \\
\hline
\end{tabular}

Se observa en este cuadro que según subsectores económicos, las pymes antioqueñas se representan en la muestra en el siguiente orden de importancia: confección (180), sustancias químicas (240), muebles de metal (360), textiles y plásticos (150 y 250). Del mismo modo, en el sector servicios están representadas las pymes dedicadas al comercio mayorista y minorista (510), el subsector de informática (720) y otras actividades (740). El muestreo por subsectores de actividad económica, junto con el tamaño, es también muy importante a la hora de estudiar la innovación en las empresas, debido a que sucede que cada región tiene una trayectoria tecnológica e histórica particular (path dependence), que hace que se especialice a lo largo del tiempo en determinadas actividades económicas, allí donde goza de ciertas ventajas comparativas y competitivas, lo 
que a su vez hace que el dinamismo de la innovación tenga más fuerza en empresas que operan en determinados subsectores. El dinamismo innovador no depende sólo de factores internos a la empresa sino también de los patrones tecnológicos que rigen el inmediato contexto subsectorial. Antioquia tradicionalmente goza de especialización en la cadena textil-confección, productos químicos y plásticos, por encima del promedio nacional.

Pasando al diseño de la encuesta y al trabajo de campo, se diseñó un cuestionario estructurado, que tuvo en cuenta el marco analítico presentado antes, lo mismo que los aportes teóricos y metodológicos ofrecidos por la primera y segunda encuestas colombianas de innovación y desarrollo tecnológico (1996/2004), la encuesta de innovación española (2003), la metodología de recolección de datos DISKO (1997-1998), el Manual de Bogotá (2000), y la última versión revisada del Manual de Oslo (2005). El periodo base del estudio fueron los años 2004-2005, y el trabajo de campo se realizó entre los meses de septiembre y octubre de 2006. En total se formularon 38 preguntas, estructuradas en los siguientes capítulos:

0 . Identificación de la empresa

1. Actividad principal

2. Atributos generales

3. Resultados de innovación en el periodo 2004-2005

4. Actividades de innovación

5 y 6 . Impactos y obstáculos de la innovación

7. Cooperación y proyectos de innovación en asocio con otras empresas e instituciones

En el estudio de caso se analizó el Comité de Empresarios para el Apoyo de la Investigación Aplicada y la Gestión Tecnológica, creado en el año 2003, gracias a la gestión de la Universidad de Antioquia y de un grupo de grandes empresarios. En el análisis se utilizaron las actas de las reuniones ordinarias del comité (40 en total), y algunas entrevistas con sus voceros. 


\subsection{Sintesis de los resultados}

La estructura empresarial antioqueña se encuentra conformada por más de cien mil empresas (101.172), de las cuales el 8\% corresponde a pymes (ver cuadro $\mathrm{N}^{\circ} 3$ ), que se concentran en su gran mayoría en Medellín y el resto del Valle de Aburrá. Desde el punto de vista del empleo, la industria aporta aproximadamente el $25,4 \%$; comercio, hoteles y restaurantes el $26,4 \%$, y los servicios sociales, comunales y personales el $23,8 \%$. La participación de la subregión del valle del Aburrá en el PIB departamental -única subregión estudiada en esta investigación- por ramas de actividad económica es la siguiente: industria manufacturera (91,2\%), comercio, restaurantes y hoteles $(70,8 \%)$ y servicios comunales, sociales y personales $(60,0 \%)$, es decir, como se afirmó en una nota anterior, el crecimiento económico y el desarrollo en Antioquia se concentra en la subregión central.

\begin{tabular}{|c|c|c|c|c|c|}
\hline \multicolumn{6}{|c|}{$\begin{array}{c}\text { CUADRo N³: DistRIBUCIÓN DE LA PYME Y GRAN EMPRESA EN ANTIOQUIA SEGÚN } \\
\text { TAMAÑO Y NÚMERO DE ESTABLECIMIENTOS } 2002 \text { y } 2006\end{array}$} \\
\hline \multirow{2}{*}{ TAMAÑo } & \multicolumn{2}{|c|}{2002} & \multicolumn{2}{|c|}{2006} & \multirow{2}{*}{ VARIACIÓN \% } \\
\hline & $\mathrm{N}$ & $\%$ & $\mathrm{~N}$ & $\%$ & \\
\hline Micro & 56.908 & 89,0 & 92.391 & 91,3 & 62,3 \\
\hline Pequeña & 5.287 & 8,3 & 6.727 & 6,6 & 27,2 \\
\hline Mediana & 982 & 1,5 & 1.580 & 1,6 & 60,9 \\
\hline Grande & 762 & 1,2 & 474 & 0,5 & $-37,8$ \\
\hline Total & 63.939 & 100 & 101.172 & 100 & 58,2 \\
\hline
\end{tabular}

En promedio, las pymes encuestadas tienen quince años de existencia y setenta y dos empleados, indicador que señala que aparte de su pequeño tamaño, las pymes de la muestra son empresas maduras según los estándares internacionales, con la experiencia suficiente para crecer mediante la realización de innovaciones. Gran parte de los empleados de estas empresas tienen niveles de formación académica que oscilan entre el bachillerato y la educación universitaria, aunque con bajos promedios de educación técnica y tecnológica. 
Otra doble característica importante de las empresas estudiadas se relaciona con el componente de capital extranjero y su participación (la de las empresas) en el mercado internacional. En el estudio se encontró que un altísimo porcentaje de las Pymes analizadas están compuestas exclusivamente por capital nacional (94.3\% promedio), y sólo un $32.9 \%$ de ellas dijo haber exportado durante el periodo 2004-2005.

Desde el punto de vista de la innovación, las empresas encuestadas se pueden definir, en un porcentaje muy significativo (94\%), como empresas innovadoras en sentido amplio, esto es, empresas cuyas innovaciones están dirigidas solamente al mercado nacional, o que son nuevas sólo para las empresas. Además, de acuerdo al grado de innovación, las innovaciones realizadas fueron de carácter incremental (61,5\%): en producto (38\%), en proceso $(39 \%)$, en comercialización (6\%), y en organización (17\%). Estos resultados los atribuyeron las empresas a la implementación de $\mathrm{I}+\mathrm{D}$ interna en su componente de desarrollo experimental $(83,7 \%)^{17}$, a la organización del lugar de trabajo $(81.8 \%)$, a la formación del personal interna o externa (73,3\%), a la adquisición de conocimientos en el mercado externo (72,7\%), y, finalmente, a la compra de maquinaria y equipo (66,7\%). Otro indicador importante para conocer los esfuerzos reales de las pymes es el monto promedio de los recursos de presupuesto invertido en el logro de los diversos tipos de innovaciones. Las empresas del estudio invirtieron en promedio: en innovación de producto (25\%), en innovación de proceso (20,6\%), en innovación de comercialización (5,8\%), y en innovación organizacional $(31,1 \%)$. Como se observa el porcentaje invertido no se correlaciona siempre con el resultado obtenido, porque, por ejemplo, el $38 \%$ de las innovaciones de producto se lograron apenas

17 Observación que hace pensar en las dificultades que este factor puede acarrear a la modernización tecnológica e, incluso, a la innovación. Dato sorprendente éste, debido a que el $65 \%$ de la empresas afirmaron haber realizado I $+D$ interna, durante el periodo de estudio considerado. Sin embargo, si se tiene en cuenta que dentro de la $I+D$, el desarrollo experimental es uno de sus componentes importantes, entonces se puede concluir que una fuerza de trabajo experimentada puede muy bien asumir este tipo de actividad de innovación, aunque de forma empírica e informal. 
con un $25 \%$ de recursos, y algo parecido acontece con la relación entre innovaciones de proceso y el monto de la inversión, es decir, la relación coste/beneficio es muy favorable respecto a estos dos tipos de innovaciones. Cosa contraria sucede con la misma relación cuando se observa la innovación organizacional, pues mientras el beneficio representó el 17\%, el monto invertido para su consecución fue cerca del $31 \%$ (casi el doble). En el caso de las innovaciones en comercialización la relación coste/beneficio resultó equilibrada (5,8\% y $6 \%$ respectivamente). En el estudio no se indagó sobre las posibles causas de estas relaciones, aunque teóricamente cabría suponer que las productividad total de los factores es mucho mayor en las innovaciones de producto y de proceso.

Debido a que la innovación es una estrategia competitiva muy riesgosa, las empresas sólo se arriesgarán a innovar si tienen una expectativa favorable sobre los impactos de las innovaciones que emprenden sobre diversos aspectos de la empresa y de sus negocios. Los datos indican que las pymes entrevistadas han aprendido a valorar el hecho de innovar, quizás debido a que por su madurez han desarrollado procesos de aprendizaje en este sentido. Así se constata por el porcentaje de empresas que afirmaron haber innovado en el periodo de estudio, encontrándose que $42,4 \%$ dijeron que la importancia de su principal innovación para el volumen de negocios de la empresa había sido muy alta, 38\% alta, y sólo un 6\% baja, sobresaliendo en este sentido las respuestas de las empresas de servicio. En general, existen diferencias significativas cuando se discrimina a las empresas por subsectores económicos, por ejemplo, las empresas del subsector de bebidas y alimentos le atribuyen una importancia muy alta a sus innovaciones $(66,7 \%)$, mientras que para las empresas del subsector de preparados de cuero y artículos de cuero apenas les atribuyen una importancia alta (50\%). En promedio, por subsectores de actividad económica, los resultados para esta variable fueron los siguientes: $42,4 \%$ de las empresas afirmaron que sus innovaciones habían tenido un impacto muy alto, $38 \%$ que alto, $11 \%$ que medio, $6 \%$ que bajo y, finalmente, $3 \%$ dijeron que el impacto de sus innovaciones había sido muy bajo. 
Discriminadas las empresas por el contenido del impacto de sus innovaciones, en promedio, el $47 \%$ de las empresas afirmaron que habían aumentado la oferta y calidad de los bienes y servicios ofrecidos, lo que les reportó en la misma proporción un aumento de su cuota de mercado. Concomitantemente, $27 \%$ de las empresas le contaron a los encuestadores que los principales impactos habían sido aumentos en la capacidad y flexibilidad de la producción, en la reducción de los costes laborales, así como en los de materias primas y energía.

Las fuentes de ideas para la innovación, distintas a $I+D$, que utilizaron las empresas en el periodo 2004-2005, fueron en su orden de importancia las siguientes: consulta con proveedores de equipos, materiales y componentes $(77,3 \%)$; consulta con empleados de las empresas $(69,7 \%)$; revistas profesionales y otros medios de información y comunicación (65,2\%); visitas a congresos, reuniones, ferias y exposiciones $(62,1 \%)$; y, las ideas aportadas por los competidores y otras empresas de la misma rama de actividad $(62,1 \%)$. Sobresale, por otra parte, la escasa significación que tuvieron para la innovación de las empresas, los aportes de las universidades y otros institutos de enseñanza (22,7\%), lo mismo que los de otros organismos públicos de investigación y centros de desarrollo tecnológico $(15,2 \%)$, lo que viene a indicar el alejamiento crítico entre las pymes y las instituciones de conocimiento y formación en Antioquia, circunstancia que seguramente esté implicando la imposibilidad de crear ventajas competitivas sostenibles que sólo se pueden fundar en innovaciones radicales, apoyadas en un predominio de I+D.

En cuanto a los obstáculos para la innovación las empresas reportaron en su orden, los siguientes: costos demasiado altos $(19,7 \%)$, riesgos económicos excesivos $(15,9 \%)$, falta de personal calificado $(24,2 \%)$, dificultades de acceso a fuentes apropiadas de financiación, y con valores menos significativos, la rigidez en la organización de las empresas, insuficiente información tecnológica y de mercados, normas y reglamentos rígidos y desinterés de los clientes por nuevos bienes y servicios. Sin embargo, puestos los 
empresarios a calificar la importancia de estos obstáculos en una escala de 1 a 5 (donde 5 como máxima calificación equivalía a un obstáculo con una importancia muy alta, 4 alta, 3 media, 2 baja y 1 muy baja), en promedio, la mayor calificación asignada fue 3 , es decir, importancia media, referida al obstáculo "costos demasiado altos", y la menor calificación 1.8 (importancia muy baja), referida al obstáculo "desinterés de los clientes por nuevos bienes y servicios". Esto significa que a pesar de que los empresarios experimentaron diversos obstáculos a la hora de emprender actividades de innovación y de obtener resultados, estos fueron valorados en un rango de importancia entre media y muy baja por los empresarios, es decir, que ninguno de los obstáculos enlistados representó una dificultad en verdad insuperable, esto es, que hubiera impedido seriamente la dinámica innovadora de las empresas. Lo anterior concuerda con lo que se señaló más arriba en este artículo, a saber, que prácticamente la totalidad de las empresas de la muestra (94\%) innovó en el periodo 2004-2005 o, lo que es lo mismo, que no encontraron obstáculos prominentes que salvar.

Entrando al tema del papel de los vínculos y relaciones de cooperación en la actividad innovadora de las empresas bajo estudio, cabe recordar, como se dijo en la nota 10, que es necesario distinguir entre relaciones empresa-universidad, vínculos empresa-universidad y cooperación empresa-universidad, entendidas como las tres etapas que sigue la evolución del contenido de las relaciones entre las empresas y las universidades. Esta triple distinción es útil teóricamente porque permite discriminar entre mecanismos de coordinación de mercado en función de la innovación y mecanismos sociales como las redes de innovación, que son redes de cooperación, basadas en la confianza y en las normas. Mientras los mecanismos de mercado posibilitan la transferencia de conocimiento de las universidades a las empresas o entre las empresas mismas, las relaciones de cooperación permiten el intercambio de conocimientos interinstitucionales, sin que los beneficios comerciales tengan que ser necesariamente la motivación inmediata que lleva a los actores a relacionarse. 
En el trabajo de campo se tuvo buen cuidado de que los empresarios comprendieran estas distinciones. Su comprensión efectivamente se confirmó al parecer por el hecho de que las dos terceras partes de las Pymes (76\%), declararon no haber cooperado en proyectos de innovación con otras empresas y/o organizaciones durante el periodo de referencia, no obstante hubieran mantenido relaciones comerciales permanentes con sus proveedores $(77,3 \%)$ clientes $(63,6 \%)$ e, incluso, con sus competidores $(60,6 \%)$, como se infiere de una lectura cuidadosa de este aspecto de los resultados de la encuesta. Por otra parte, contrasta el hecho de que los vínculos con universidades, centros de formación superior y CDT, juegan un papel significativamente menor en las actividades innovadoras de las empresas, como se destacó antes. Para constatarlo, compárense los cuadros $\mathrm{N}^{\circ} 4$ y №5:

\begin{tabular}{|c|c|c|c|c|}
\hline \multicolumn{5}{|c|}{$\begin{array}{l}\text { CUADRO N }{ }^{\circ} \text { : MATRIZ DE CONECTIVIDAD GLOBAL DE LA PYME INNOVADORA DE LA } \\
\text { MUESTRA CON OTRAS EMPRESAS Y CON LAS INSTITUCIONES DE CONOCIMIENTO E } \\
\text { INFORMACIÓN (CONSOLIDADO) }\end{array}$} \\
\hline \multirow{3}{*}{ INSTITUCIONES Y/O AGENTES } & \multicolumn{4}{|c|}{ TOTAL PYME (MUESTRA) } \\
\hline & \multicolumn{2}{|c|}{ Vinculo } & \multicolumn{2}{|c|}{$\begin{array}{l}\text { \% BENEFICIO } \\
\text { (PROMEDIO) }\end{array}$} \\
\hline & $\mathbf{N}$ & $\%$ & si & NO \\
\hline \multicolumn{5}{|l|}{ EMPRESAS } \\
\hline Con proveedores & 51 & 77,3 & 91,6 & 8,4 \\
\hline Con clientes & 42 & 63,6 & 100,0 & 0,0 \\
\hline Con competidores & 40 & 60,6 & 96,0 & 4,0 \\
\hline Con otras empresas de su mismo grupo* & 4 & 6,1 & 100,0 & 0,0 \\
\hline $\begin{array}{l}\text { Entre departamentos dentro de la misma } \\
\text { empresa }\end{array}$ & 46 & 69,7 & 100,0 & 0,0 \\
\hline Con otras empresas ** & 41 & 62,1 & 95,1 & 4,9 \\
\hline \multicolumn{5}{|c|}{ INSTITUCIONES DE CONOCIMIENTO E INFORMACIÓN } \\
\hline $\begin{array}{l}\text { Con universidades u otros institutos de } \\
\text { enseñanza superior }\end{array}$ & 14 & 21,2 & 95,0 & 5,0 \\
\hline $\begin{array}{l}\text { Con organismos públicos de investigación o } \\
\text { Centros de Desarrollo Tecnológico }\end{array}$ & 10 & 15,1 & 95,0 & 5,0 \\
\hline Con otras fuentes de información externa ${ }^{\star \star *}$ & 43 & 65,1 & 96,4 & 3,6 \\
\hline \multicolumn{5}{|c|}{$\begin{array}{ll}* & \text { Si aplica } \\
* * & \text { En congresos, reuniones, ferias, exposiciones } \\
* * * & \text { Revistas profesionales, sitios web, prensa, documentación en general }\end{array}$} \\
\hline \multicolumn{5}{|c|}{ Fuente: Encuesta CECM-ITM (2006). Base: 66/70 empresas } \\
\hline
\end{tabular}


En el cuadro $\mathrm{N}^{\circ} 4$ se presenta una matriz de conectividad en donde se establecen indicadores de vínculos no-cooperativos (según se explicó antes) entre las pymes del estudio y otras empresas e instituciones de conocimiento e información, que constituyen una clave muy importante para entender la estructura y dinámica de un sistema de innovación regional. En él se observa, por una parte, numerosos y fuertes vínculos entre las pymes y una amplia variedad de ámbitos empresariales, que constituyen encadenamientos de innovación y, por otra parte, la debilidad de los vínculos entre las pymes y las instituciones del conocimiento, que según la literatura especializada son una condición sine qua non para una estrategia competitiva sustentada en la innovación por I+D.

\begin{tabular}{|c|c|c|c|c|c|c|}
\hline \multicolumn{7}{|c|}{$\begin{array}{l}\text { CUADRO } N^{\circ} 5: \text { MATRIZ DE CONECTIVIDAD SEGÜN TAMAÑO DE EMPRESA, } \\
\text { AGENTE COOPERANTE Y ÁMBITO GEOGRÁFICO DEL COOPERANTE }\end{array}$} \\
\hline \multirow{4}{*}{ AGENTES COOPERANTES } & \multicolumn{6}{|c|}{ TAMAÑO DE EMPRESA } \\
\hline & \multirow{2}{*}{\multicolumn{2}{|c|}{$\begin{array}{l}\text { PEQUEÑA } \\
\text { IEDR }_{1}\end{array}$}} & \multirow{2}{*}{\multicolumn{2}{|c|}{$\frac{\text { MEDIANA }}{\text { IEDR }_{2}}$}} & \multirow{2}{*}{\multicolumn{2}{|c|}{ IGDR }} \\
\hline & & & & & & \\
\hline & $\mathrm{N}$ & $\%$ & $\mathrm{~N}$ & $\%$ & $\mathrm{~N}$ & $\%$ \\
\hline \multicolumn{7}{|c|}{ LOCALES } \\
\hline Empresas & 2 & 14,3 & 0 & 0,0 & 2 & 12,5 \\
\hline Universidades* & 2 & 14,3 & 0 & 0,0 & 2 & 12,5 \\
\hline CDT & 0 & 0,0 & 0 & 0,0 & 0 & 0.0 \\
\hline Otras Instituciones ${ }^{* *}$ & 1 & 7,1 & 0 & 0,0 & 1 & 6,2 \\
\hline \multicolumn{7}{|l|}{ Regionales } \\
\hline Empresas & 1 & 7,1 & 1 & 7,1 & 2 & 12,5 \\
\hline Universidades & 2 & 14,3 & 1 & 50,0 & 3 & 18,8 \\
\hline CDT & 1 & 7,1 & 0 & 0,0 & 1 & 6,2 \\
\hline Otras Instituciones & 0 & 0,0 & 0 & 0,0 & 0 & 0,0 \\
\hline \multicolumn{7}{|c|}{ NACIONALES } \\
\hline Empresas & 6 & 42,9 & 2 & 100,0 & 8 & 50,0 \\
\hline Universidades & 0 & 0,0 & 0 & 0,0 & 0 & 0,0 \\
\hline CDT & 0 & 0,0 & 0 & 0,0 & 0 & 0,0 \\
\hline Otras Instituciones & 0 & 0,0 & 0 & 0,0 & 0 & 0,0 \\
\hline \multicolumn{7}{|c|}{ INTERNACIONALES } \\
\hline Empresas & 3 & 21,4 & 0 & 0,0 & 3 & 21,4 \\
\hline Universidades & 0 & 0,0 & 0 & 0,0 & 0 & 0,0 \\
\hline CDT & 0 & 0,0 & 0 & 0,0 & 0 & 0,0 \\
\hline Otras Instituciones & 1 & 7,1 & 0 & 0,0 & 1 & 6,2 \\
\hline Total & \multicolumn{2}{|c|}{ NTotal: } & & $\begin{array}{l}\text { otal: } \\
2\end{array}$ & \multicolumn{2}{|c|}{$\begin{array}{c}\text { total: } \\
16\end{array}$} \\
\hline \multicolumn{7}{|c|}{$\begin{array}{l}\text { * Grupos y Centros de Investigación } \\
* * \text { Gubernamentales locales, regionales y nacionales; gremiales; Cámaras de } \\
\text { Comercio; Sindicatos; Fundaciones, Cooperativas; ONG; instituciones de educación } \\
\text { técnica-tecnológica }\end{array}$} \\
\hline \multicolumn{7}{|c|}{ Fuente: Encuesta CECM-ITM (2006) } \\
\hline
\end{tabular}


En el cuadro $\mathrm{N}^{\circ} 5$, por su parte, prácticamente se muestra la debilidad de una cultura de cooperación para la innovación, ya que sólo un $24 \%$ de las empresas mantuvieron lazos de cooperación en 2004-2005, con otras empresas e instituciones. Este hecho se hace más palpable cuando el $50 \%$ de dichas empresas afirmaron que era la primera vez que lo habían hecho ${ }^{18}$. Entre tanto, para el restante $50 \%$, la cooperación ya hacia parte de sus estrategias de competitividad. Sin duda alguna, estos hallazgos deben ser profundizados, sobre todo el hecho de que las empresas de servicios hayan sido más dinámicas y asertivas en el tema, si se les compara con las empresas manufactureras. Otra observación que debe tenerse en cuenta es que el $86 \%$ de las empresas que no cooperaron argumentaron como razón principal no haber tenido necesidad de hacerlo. Según el ámbito geográfico y el tipo de agente con el cual se cooperó, se destacan el ámbito nacional de los cooperantes, y las empresas como los principales colaboradores. Los ámbitos local y regional, y las universidades, CDT y otras organizaciones sociales, sólo fueron destacados por aquellas pocas empresas cuyos mercados principales se ubican en el entorno local. No obstante, las pocas empresas que dijeron haber cooperado con este tipo de agentes les atribuyeron un alto nivel de importancia.

Por último, en breve, el estudio de caso sobre la estructura de interfaz mostró, por su parte, que la colaboración empresa-universidad $y$, con menos frecuencia, con otras instituciones políticas y sociales, constituye hoy por hoy un núcleo "duro" alrededor del cual, o por efectos de su multiplicación, la conformación de redes de innovación como redes de conocimiento puede llegar a convertirse en el mediano plazo en el punto de quiebre de una trayectoria histórica esquiva a la cooperación, que hasta hoy ha caracterizado a los sectores empresariales y a las instituciones del conocimiento, por no hablar de un alejamiento más pertinaz aún de 18 Un estudio reciente muy relevante en este aspecto llega a las mismas conclusiones
(Hincapié: 2004). 
los gobiernos y otros sectores sociales. Las relaciones de confianza logradas en los últimos cuatro o cinco años entre los actores así como los resultados de innovación alcanzados, dejan ver a las claras como la cooperación en I+D entre empresas e instituciones del conocimiento (universidades, instituciones técnicas y tecnológicas de formación, CDT, etc.), viene emergido como una estrategia de innovación y desarrollo empresarial en un momento en que la región se apresta a entrar en diversos tratados de libre comercio y, por lo tanto, a globalizar su economía y por ende su sistema de innovación. Sin embargo, en este proceso es muy notorio el escaso papel que aún juegan las pymes, ya que en número insignificante han logrado entrar en esta dinámica. En efecto, mientras que el $32 \%$ de los miembros activos de dicho comité lo constituyen las grandes empresas, un bajísimo porcentaje, apenas un $5 \%$, corresponde a pymes. Esta baja participación parece concordar con los datos del estudio por encuestas, pues del $25 \%$ del total de las pymes que dijeron haber cooperado con otros agentes en proyectos de innovación y desarrollo en el periodo $2004-2005$, sólo un $33 \%$ de las empresas afirmó haberlo hecho con universidades y otras instituciones de investigación y conocimiento como CDT y grupos de investigación; empresas cuyos mercados no alcanzan siquiera la dimensión nacional, y están constreñidas a los más estrechos e inmóviles mercados local y regional.

\section{Conclusión}

Tanto la aplicación de una encuesta a una muestra de setenta pequeñas y medianas empresas antioqueñas, como el estudio de caso de una estructura de interfaz universidad-empresa-estado, han puesto de presente dos hechos importantes: el primero, que en su gran mayoría las pymes en Antioquia desarrollan actividades de innovación y obtienen resultados favorables, aunque con un componente de I+D débil, lo que permite definirlas como empresas innovadoras en sentido amplio. En este sentido los resultados obtenidos en este estudio son muy semejantes a los hallazgos de 
la primera encuesta nacional de innovación y desarrollo tecnológico que se aplicó en Colombia hace una década, lo que permite concluir que el panorama de la innovación en Colombia, al menos en lo que toca a las pymes, no ha cambiado sustancialmente. Por otra parte, y en segundo lugar, si se toma en cuenta el mismo referente de la primera encuesta nacional, el surgimiento y consolidación en la región de un Comité Universidad-Empresa-Estado, en el último lustro - que dicho sea de paso se ha replicado en experiencias similares en otras regiones colombianas-, se revela como una auténtica innovación social e institucional, lo que permite tener buenas expectativas en que este tipo de mecanismo puede dar lugar a fuertes y diversificadas relaciones de cooperación para la innovación, es decir, que se pueden constituir redes de innovación como redes de conocimiento, pasibles de robustecer el sistema de innovación. Sin embargo, el papel que puedan jugar en el mediano plazo las pymes en esta clase de mecanismo no parece alentador a la luz de los hallazgos realizados en este estudio.

Habrá que esperar qué resulta de la segunda parte de este trabajo, pues seguramente podrá dar lugar a un mejor conocimiento de este aspecto particular de las pymes, para poder tener un mejor diagnóstico sobre si se mantendrán en la inercia de su aislamiento proverbial, o si, por el contrario, se atreverán a replicar la lección acaecida en otras latitudes del mundo en desarrollo, en donde la cooperación para la innovación y la competitividad no sólo ha fortalecido al sector de la pymes sino que, además, ha traído grandes beneficios económicos y sociales a las regiones donde estas se encuentran localizadas. 


\section{Bibliografía}

ANDERSSON, M. y KARLSSON Ch. (2004), "Regional Innovation Systems in Small \& Medium-Sized Regions. A critical Review \& Assessment." En: CESIS Electronic Working Paper Series. [En línea] $\mathrm{N}^{\circ} 10$, Agosto 2004, disponible en: http://www.infra.kth.se/cesis/documents/WP10.pdf

ÁNGEL DEL BRÍO, J., FERNÁNDEZ, E. y JUNQUERA, B. (2006), “QQué preocupa de la cooperación en $\mathrm{I}+\mathrm{D}+\mathrm{i}$ ? : Un análisis del período 1996 2005" en Revista Madrid. [En línea] N 36, Mayo - Junio 2006, disponible en: http://www.madrimasd.org/revista/revista36/tribuna/tribuna2.asp

AROCENA, R. y SUTZ, J. (1999), "Mirando los Sistemas Nacionales de Innovación desde el Sur" en $O E I$ [En línea] disponible en: http://www.oei.es/salactsi/sutzarcena.htm

.(2002), "Innovation Systems and Developing Countries", DRUID (Danish Research Unit for Industrial Dynamics) Working Paper $\mathrm{N}^{\circ}$ 02-05 [En línea] disponible en: http://www.druid.dk/uploads/tx picturedb/wp02-05.pdf. Se cita de la traducción española: Sistemas de Innovación en países en desarrollo. Disponible en: Revista OEI: http:// www.oei.es/salactsi/arocenasutz.htm

ASHEIM, B. y ISAKSEN, A. (1996), Location, Agglomeration and Innovation: Towards Regional Innovation Systems in Norway, STEP Report R-13, Oslo.

(2001), "Los sistemas regionales de innovación, las PYMES y la política de innovación", Sistemas Regionales de Innovación, (Ed.). Olazaran M. et Gómez Uranga M. UPV-EHU, Zarauz.

CAMISÓN C. et al., (2003), "Marco conceptual de la relación entre innovación y tamaño organizativo" en Revista Madritd. [En línea] $\mathrm{N}^{\circ} 19$, Octubre - Noviembre 2003, disponible en: http://www.madrimasd.org/ revista/revista19/tribuna/tribuna1.asp

BORONAT M. y VILLAR, A. (2005), "Innovando a través del establecimiento de alianzas estratégicas: La generación de competencias distintivas en conocimiento y su efecto en el desempeño organizativo, en Revista Madri+d. [En línea] No 36, Mayo - Junio 2006, disponible en: http://www.madrimasd.org/revista/revista36/tribuna/tribuna1.asp

CARLSON, B. y STANKIEWICZ, R. (1991), "On the nature, function and composition of technological systems", Journal of Evolutionary Economics, 1, 93-118 
(2002), "Innovation systems: analytical and methodological issues", Research Policy 31 (2002) 233-245.

CASANUEVA, C.; CASTRO, I. y GALÁN J., (2006), "Capital Social, confianza e innovación. El caso de un sistema productivo local tradicional" en Revista Madri+d. [En línea] No 36, mayo - junio 2006, disponible en: http:/www.madrimasd.org/revista/revista36/tribuna/tribuna4.asp

CASAS R., et al., (2001), La Formación de Redes de Conocimiento. Una perspectiva regional desde México. Primera Edición. Barcelona. Anthropos.

CASTELLS, Manuel. (1999), La era de la información Vol. I y II. Economía Sociedad y Cultura. Siglo XXI. México.

COOKE, P.; URANGA, M.G. y ETXEBARRIA, G. (1998), "Regional Systems of Innovation: An Evolutionary Perspective. Environment and Planning" A 30: 1563-1584.

(1997), “Regional Innovation Systems: Institutional and Organizational Dimensions”, Research Policy, 26, 475-491.

EDQUIST, C. (Ed.) (1997), "Systems of innovation. Technologies, Institutions and Organizations". London: Pinter Publishers.

ERIKSSON, A. (2000) Regional Innovation System - Från Teori till Genomförande (Regional Innovation Systems - from theory to accomplishment), Swedish Office of Science and Technology, Stockholm.

FRITSCH M. (2004), "Cooperation and the efficiency of regional R\&D activities" en Cambridge Journal of Economics, [En línea] Vol. 28, No. 6, 2004, disponible en:http://cje.oxfordjournals.org/cgi/content/abstract/28/6/829

IORIO, Roberto (2003), “A synthetic view on networks, social capital, university-industry. Collaboration and policy instruments for research collaborations" [En línea] disponible en: http://www.euintangibles.net/library/ localfiles/WP3/3.7_Iorio_2003.pdf. Se cita de la traducción en español, elaborada por Gabriel Cataño Rojas. 2007.

JARAMILLO, H; LUGONES G. y SALAZAR, M. (2000), Manual de Bogotá. Normalización de Indicadores de Innovación Tecnológica en América Latina y el Caribe. Bogotá.

KOSCHATZKY, K. (1998a), "Innovation Networks of Industry and Business-Related Services - Relations between Innovation Intensity of Firms and Regional Inter-firm Cooperation", European Planning Studies, 7, pp. 737-757.

(1998b), "Firm Innovation and Region: the Role of Space in Innovation Processes", International Journal of Innovation Management, Vol. 2 No. 4, pp. 383-408. 
(2001), "Networks of Innovation Research and Innovation Policy". En: Koschatzky et al. (2001): "Innovation Networks: concepts and challenges in the European perspective". Physica-Verlag, Heidelberg.

LUNA, M. y VELASCO, J. (2005), "Redes de conocimiento: principios de coordinación y mecanismos de integración" Seminario Redes de conocimiento como nueva forma de creación colaborativa: su construcción, dinámica y gestión, 24 y 25 de noviembre de 2005, Buenos Aires, RICYT-CYTED.

LUNDVALL, B. (1995), "Introduction", in Lundvall, B. (ed) (1995), National Systems of Innovation - Towards a Theory of Innovation and Interactive Learning, Biddles Ltd, London.

MARTÍNEZ DEL RÍO, J., y CÉSPEDES-LLORENTE J. (2006), "Generación y difusión de la innovación en distritos industriales" en Revista Madritd [En línea] No 36. Mayo -Junio 2006. Universidad de Almería, disponible en: http://www.madrimasd.org/revista/revista36/editorial/ editorial.asp]

MEEUS, M., OERLEMANS, L. y VAN DIJCK, J. (1999), "Regional Systems of Innovation from within - an Empirical Specification of the Relation Between Technological Dynamics and Interaction Between Multiple Actors in a Dutch Region”, ECIS Working Paper No. 99.1.

MORGAN, K. (1997), "The learning region: institutions, innovation and regional renewal". Regional Studies, vol. 31, n 5, pp. 491-503.

NOISI, J. (2000), "The internalization of industrial R\&D: From technology transfer to the learning organization", Research Policy, 28, pp. 107-117.

OECD (2005), Oslo Manual. "The measurement of scientific and technological activities. Proposed guidelines for collecting and interpreting innovation data", Paris.

PÉREZ, C. (1988), en M.Pulido ed., Venezuela: Desafios y Propuestas, UcabSic, Caracas, pp.63-109.

PÉREZ, C. (1999), Revista del Banco Central de Venezuela, Año XIII, No. 2, pp. 14-29.

ROZGA R., (2003), "Sistemas Regionales de Innovación: Antecedentes, Origen y Perspectivas" en Convergencia, Revista de Ciencias Sociales. $\mathrm{N}^{\circ} 33$, Año 10, septiembre -diciembre 2003, pp.225-246.

SEBASTIÁN, J., (2000a),"Las Redes de Cooperación como modelo organizativo y funcional para la I+D" en Redes. Vol.7. $N^{\circ} 15$, pp. 97-111. 
(2000b),"La Cultura de la Cooperación en la I+D+i" en Espacios. [En línea] Vol. 21 (2) 2000 disponible en: http://www.revistaespacios.com/a00v21n02/80002102.html

(2000c), "Percepciones sobre la cooperación Académica y Científica entre España y América Latina" disponible en: http://www. campus-oei.org/superior/boletin2e.htm\#a

(2000d),"Tendencias en la Cooperación entre las universidades y las empresas" en Ciecas. Centro de Investigaciones Económicas, Administrativas y Sociales del Instituto Politécnico Nacional. México, disponible en http://www.oei.es/cursoctsi/uruguay/ciecas.pdf

SCHUMPETER, J. (1934), "Theory of Economic Development". Harvard University Press, Cambridge, Mass.

YIN, R.K. (1994), "Case study research. Design and methods". Sage Publications. 